\section{Profound associations between pain and quality of life}

\section{Stephen Thielke \\ Department of Psychiatry and Behavioral Sciences, University of Washington, WA, USA}

Since researchers began scrutinizing the phenomenology of pain about 40 years ago, it has become increasingly evident that pain is not experienced, and cannot be studied, in isolation. It is not just a symptom. Pain changes how people behave, think, and interact with others. A key advance was differentiating the different aspects of the experience of pain: injury, nociception, affective experience, and pain behaviour. ${ }^{1}$ Novel theoretical approaches, which grew out of models of health psychology, encouraged a nuanced, holistic conception of pain, and an understanding of how pain related to the individual's activities, relationships, and quality of life.

At the same time, it has become obvious that much of the clinical care provided for pain has considered it as a straightforward symptom and has applied a largely medicalized model in managing it. The movement to treat pain as a fifth vital sign, the most visible clinical revolution of the last dozen years, assumed that by measuring pain in one dimension (a zero to ten scale), clinicians could select appropriate treatment, usually medications. This model is widely considered a failure, and has not only failed to lower rates of chronic pain, but may have fueled increases in opioid prescriptions. ${ }^{2}$ An Institute of Medicine (IOM) report from 2011, Relieving Pain in America: A Blueprint for Transforming Prevention, Care, Education and Research, testifies to the pervasive shortcomings in the understanding of and holistic treatment for pain, and the need for new clinical approaches. ${ }^{3}$

The discipline of health psychology has always taken a broad, holistic approach to understanding human experiences and behaviors, and has become a major force in rethinking pain. This special edition of Health Psychology Research, focused on pain and quality of life, advances this body of work in several ways: it delineates the historical traditions of the field; it deepens the understanding of pain's broad effects on well-being; it refines the tools for assessing pain; it ascertains what therapeutic modalities work; and it maps out future research. More generally, it inspires hope that - with the right scientific rigor and clinical acumen - pain treatment can indeed be transformed in ways that will enhance patients' subjective wellbeing.

A perfect way to start this mission is in the perspectives of Professor Robert Cummins, an expert on quality of life research. Dr
Theofilou's interview with him contains an entertaining and enlightening account of the history of the discipline, as well as the core principles that undergird it, and how pain and subjective well-being relate. Professor Cummins clears up some common misconceptions about quality of life research, and illustrates how important it has been and will remain for health psychology, psychosocial research, pain research, and clinical care.

The article by Dedeli and Kaptan Spiritual concerns in pain management, is proof of how attending to quality of life from the patient's perspective yields many useful clinical insights. They broadly review spirituality and pain, and find numerous areas of overlap, as well as topics that require more research. Clinicians often ignore patients' spirituality, despite the fact that many patients rely on spiritual belief systems as a way to make sense of, discuss, and deal with pain. I encourage any clinician who treats patients who have pain to read this article.

More direct evidence for the significance of quality of life research in health psychology comes in the work of Welhelmson, Fritzell, Eklund, and Dahlin-Ivanoff. Their groundbreaking research assessed life satisfaction among older adults who had different degrees of frailty. Although their work found that frailty was associated with decreased life satisfaction, they also found that older adults' satisfaction with life as a whole was almost as high as in younger age groups. This both helps to challenge a common stereotype about aging (that older adults' quality of life declines) and illustrates ways to prolong and maximize life satisfaction.

A review of the book Advanced Cancer: Pain and Quality of Life (Chow and Merrick, editors; review by Dr Theofilou) develops several of the themes which Dr Cummins discussed, and highlights recent research. Cancer pain is more than just a symptom of a potentially terminal disease: it also has meaning for patients and providers, and this book bolsters the biopsychosocial approach to patients which health psychology has championed.

Research by Protopapa and Senior, Does pain acceptance predict physical and psychological outcomes in cancer outpatients with pain?, addresses this question of the meaning of pain in some detail. Using a sample of outpatients from an oncology center, they measured pain acceptance and ascertained its association with physical and psychological outcomes. They found that pain acceptance was an important predictor of functional outcomes, even after controlling for disease-related variables. These findings encourage additional trials to enhance pain acceptance for patients with cancer pain, in particular those focused on activity engagement.

A study protocol by Theofilou, Aroni, Tsironi
Correspondence: Stephen Thielke, Department of Psychiatry and Behavioral Sciences, VA Puget Sound Health Care System - VAPSHCS, $1660 \mathrm{~S}$. Columbian Way, Box 358280, Seattle 98108, WA, USA.

Tel. +1.206.764.2815 - Fax: +.206 .764 .2569$

E-mail: sthielke@u.washington.edu

Received for publication: 23 September 2013. Accepted for publication: 23 September 2013.

This work is licensed under a Creative Commons Attribution NonCommercial 3.0 License (CC BYNC 3.0).

(C) Copyright S. Thielke, 2013

Licensee PAGEPress, Italy

Health Psychology Research 2013; 1:e36 doi:10.4082/hpr.2013.e36

and Zyga outlines how pain self-efficacy and quality of life will be measured in a study of hemodialysis patients. These results will sharpen the understanding of how the domains of health, quality of life, pain experience, and pain coping are interrelated, and will identify which of them might be most amenable to clinical interventions.

Both clinicians and researchers have come to appreciate that, just as pain is a multifaceted phenomenon that cannot be measured simply and that has many effects on quality of life, so simple interventions are unlikely to produce much benefit. Sexton-Radek and Chami's case study of a successful case of collaboration in a pain clinic is a direct illustration of how health psychological approaches can enhance patient outcomes in real-world treatment settings.

Two carefully conducted intervention studies, grounded in health psychology, achieved their results by formulating pain as an experience that affects the whole person. Research by Wonders, Whisler, Loy, et al. found that a home-based exercise program improved symptoms of chemotherapy-induced peripheral neuropathy in breast cancer patients. This intervention, although preliminary, is a creative approach to a common pain syndrome that has significant effects on quality of life. Research by Steiner, Bogusch, and Bigatti applied Acceptance and Commitment Therapy (ACT) in patients with fibromyalgia. Fibromyalgia is often a significant challenge to treat, and this study's positive results point the way to new approaches for fibromyalgia and other apparently intractable types of pain.

Finally, Megari's paper is a very important contribution to this special edition. She studied thoroughly the literature with regards to quality of life and especially health-related quality of life in chronic diseases. Quality of life is inherently a dynamic, multi-level and 
complex concept, reflecting objective, subjective, macro-societal, and micro-individual, positive and negative influences which interact.

All of the articles in this edition grow out of the deep conviction that people who experience pain cannot be understood unless pain in seen through the lens of quality of life, and vice versa. As Professor Cummins noted more succinctly, pain has a powerful ability to defeat subjective well-being homeostasis. In closing, I echo Professor Cummins's rallying cry for cross-disciplinary collaboration, which is the best way both to understand pain and quality of life, and in the process to reduce the former and enhance the latter.

I would like to express sincere thanks to the authors for sharing their outstanding research, and to Dr. Paraskevi Theofilou for inviting me to edit this special edition.

\section{References}

1. Wall PD. On the relation of injury to pain.
The John J. Bonica lecture. Pain 1979;6: 253-64.

2. Thielke SM, Simoni-Wastila L, Edlund MJ, et al. Age and sex trends in long-term opioid use in two large American health systems between 2000 and 2005. Pain Med 2010;11:248-56.

3. IOM (Institute of Medicine). Relieving pain in America: a blueprint for transforming prevention, care, education, and research. Washington, DC: The National Academies Press; 2011. 